analysis showed a normal number of $\mathrm{B}$ cells but a decrease in naive and memory CD4 T cells upon TNF $\alpha$ blockade. Within the $\mathrm{B}$ cell population, TNF blockade significantly increased the frequency of memory $B$, which displayed an activated phenotype with increased expression of CD40 and HLA-DR. In parallel, however, TNF blockade decreased the frequency of CD138 plasmablasts, suggesting a defective maturation towards antibody-producing cells. Moreover, TNF blockade significantly decreased the degree of somatic hypermutation as evidenced by Ig $\kappa$ REHMA analysis of peripheral blood $B$ cells before and after treatment.

Conclusion TNF blockade severely impairs TD humoural responses by interfering with the affinity maturation and differentiation of activated $B$ cells towards antibody producing cells.

\title{
A32 TNF $\alpha$ BLOCKADE IMPAIRS T CELL DEPENDENT HUMOURAL RESPONSES
}

G F Salinas, L De Rycke, T Cantaert, M van de Burg, B Barendregt, P Remans, P Peter Tak, D Baeten Clinical Immunology and Rheumatology, Academical Medical Centrum/ Department of Immunology, Erasmus Medical Center, Rotterdam, The Netherlands

GFS and LDeR contributed equally to this work

10.1136/ard.2010.129585f

Objective Tumour necrosis factor $\alpha$ (TNF $\alpha$ ) blockade in spondyloarthritis ( $\mathrm{SpA}$ ) induces antibodies specific for double stranded DNA, which is a $T$ cell independent (TI) antigen. As these antibodies were restricted to the IgM isotype and no antibodies to $T$ cell dependent (TD) antigens were induced, we investigated here if TNF blockade impairs the induction and maturation of TD humoural responses.

Methods $30 \mathrm{SpA}$ patients (20 treated with TNF $\alpha$ blockade, 10 untreated controls) were vaccinated with a TD vaccine to hepatitis B and a TI vaccine to $S$ pneumoniae. Another $10 \mathrm{SpA}$ patients treated with infliximab were vaccinated with a TD vaccine to $S$ pneumoniae. Serum and peripheral blood mononuclear cells were collected before and after vaccination. Vaccine-specific antibody titres were measured by ELISA. $\mathrm{B}$ and $\mathrm{T}$ cell populations were evaluated by flow cytometry. Somatic hypermutation was determined by the Igк REHMA assay (Andersen, Blood 2005).

Results IgM and IgG responses against TI antigens were moderately decreased in anti-TNF $\alpha$ treated patients compared to controls but were still robust. In contrast, $\operatorname{Ig} G$ responses against TD antigens were almost completely absent in treated patients. The greater suppression of TD versus TI responses by TNF $\alpha$ blockade was confirmed by lower IgG titres with TD versus TI vaccines against anti-S pneumoniae. Phenotypic 